\title{
R-S learning in brain damaged and control subjects
}

CHARLES V. LAIR, DONALD H. KAUSLER AND CARROLL W. HARPENAU

SAINT LOUIS UNIVERSITY

Brain damaged $S_{s}$ displayed less symmetry in paired-associate learning than control $S s$ as measured by both absolute and relative $R-S$ recall. This decrease in symmetry was apparent whether or not a marked deficiency in S-R learning was simultaneously present.

Ekstrand (1966) noted "that the difference between forward and backward associative learning has been drastically overestimated and that if symmetry is not the rule, asymmetry will be very small (p. 60). "Near symmetry of forward (S-R) and backward (R-S) learning is especially apparent when both $S$ and $R$ pairedassociate components are meaningful words and differential availability of $\mathrm{S}$ and $\mathrm{R}$ components (cf. Ekstrand, 1966) is largely eliminated. For example, $\mathrm{R}-\mathrm{S}$ learning (as measured by recall) averaged $87.7 \%$ of S-R learning in a study by Dron \& Boe (1964) with college students as Ss and $85.1 \%$ in a study by Kausler \& Lair (1965) with noncollege adults as Ss.

Ekstrand (1966) also stressed the importance of investigating the conditions under which symmetry breaks down. Contemporary research has investigated primarily task and practice variables as parameters of symmetry. However, another important direction rests in investigating symmetry as a function of $S$ variables. One such variable is the level of functioning of the central nervous system. Kausler \& Lair (1965) found that elderly Ss displayed significantly less R-S learning, in terms of both absolute and relative (to $S-R$ learning) amounts, than younger Ss, even though the two groups did not differ in S-R learning. Viewing learning deficiencies in old age as reflecting brain deterioration (e.g., Inglis, 1957, 1959) and generalizing from the Kausler and Lair findings, the possibility exists that symmetry is a learning phenomenon which diminishes whenever brain deterioration is present. The present study tested this hypothesis by contrasting the R-S performance of brain damaged and control Ss.

\section{Method}

The brain damaged group (Group $B, N=18$ ) consisted of 9 focalized and 9 generalized patients, as determined by neurological diagnosis (mean age $=42.4$ years; mean years of formal education $=10.0$ years). Diagnostic classification included cerebral hemorrhage, CBS due to alcoholism, tumor, Huntington's chorea, and encephalitis. In order to minimize motivational deficit resulting from long term hospitalization patients hospitalized for more than 3 years were excluded from this study. The control group (Group $\mathrm{C}, \mathrm{N}=18$ ) consisted of general medical patients in a medical school hospital who were without a history of alcoholism and neurological or psychiatric disorder (mean age $=39.0$ years; mean education $=11.6$ years). A t-test revealed that the two groups did not differ significantly in age or educational level.

The paired-associate list, identical to that of Kausler \& Lair (1965), contained 10 pairs of unrelated words selected from the Minnesota norms. Following practice on a two pair warm-up list, the pairs were presented by means of $3 \times 5$ index cards at a $4: 4$ sec. rate (anticipation method) for 15 trials or to a criterion of one perfect trial, whichever came first. Three different serial orders were employed. At the end of $S-R$ practice Ss were given one $\mathrm{R}-\mathrm{S}$ recall trial at a selfpaced rate. On this trial the $R$ words were presented one at a time (via index cards) in a random order, and $S$ was requested to recall the associated $S$ word. Results and Discussion

Every $S$ in Group $C$ learned the $S-R$ list to criterion within 15 trials (mean number of trials $=6.89$; $\mathrm{SD}=$ 2.49). Eight of the 9 focalized patients but none of the generalized patients learned the list to criterion (mean and SD for the focalized patients $=11.89$ and 3.66 , respectively). The maximum number of $S-R$ pairs anticipated correctly on any single trial was determined for each $\mathrm{S}$. This score provided a baseline for computing $S$ 's relative $\mathrm{R}-\mathrm{S}$ score. Group $\mathrm{C}$, of course, averaged 10 correct S-R pairs without variability; Group B had a mean of 7.50 and SD of 2.79. Although the difference between group means was statistically significant $(t=3.85$, df $=34, p<.01)$, this difference was attributable solely to the generalized patients whose mean and SD were 5.11 and 2.03 , respectively. The corresponding statistics for the focalized patients were 9.89 and 0.28 . Thus only the generalized patients were markedly below the control Ss in terms of level of $S-R$ mastery.

For absolute $R-S$ recall the mean and SD were 8.83 and 1.44 and 5.44 and 2.63 for Group C and Group B, respectively, a difference in means that was statistically significant $(t=4.84, \mathrm{df}=34, \mathrm{p}<.01)$. In view of the disparity in S-R learning between the focalized and generalized patients a separate R-S analysis was made for these two subgroups. The mean $\mathrm{R}-\mathrm{S}$ score was 7.22 for the focalized and 3.67 for the generalized patients. Both of these means were significantly less than the mean for Group $C(t=2.37, d f=25, p<.05$ and $t=6.79, d f=25$, $\mathrm{p}<.01$ ). In terms of relative $\mathrm{R}-\mathrm{S}$ scores (the ratio of $\mathrm{R}-\mathrm{S}$ recall to the maximum number of $\mathrm{S}-\mathrm{R}$ pairs correctly anticipated on a single trial) Group $C$ averaged $88.3 \%$ and Group B $72.5 \%$, a difference that was statistically significant (median test, $\chi^{2}=11.26, \mathrm{df}=1$, $\mathrm{p}<.001)$. Moreover, the depression of relative $\mathbf{R}-\mathbf{S}$ scores in Group B was independent of classificationthe focalized patients averaged $73.0 \%$ and the generalized 
$71.8 \%$. Thus brain damaged Ss displayed less symmetry than controls whether or not their S-R performance differed markedly from control S-R performance.

\section{References}

Dron, D. N., \& Boe, E. E. R-S paired-associate learning as a function of percentage occurrence of response members. $J$. verbal Learn. verbal Behav., 1964, 3, 130-131.
Ekstrand, B. R. Backward associations. Psychol. Bull., 1966, 65, 50-64.

Inglis, J. An experimental study of learning and "memory function" in elderly psychiatric patients. J. ment. Sci., 1957, 103, 796-893. Inglis, J. A paired associate learning test for use with elderly psychiatric patients. J. ment. Sci., 1959, 105, 440-443.

Kausler, D. H., \& Lair, C. V. R-S ("backward") paired-associate learning in elderly subjects. J. Gerontol., 1965, 20, 29-31. 